\author{
Qingxiang Zhou ${ }^{1,2}$ \\ Jingfu Liu ${ }^{1}$ \\ Guibin Jiang 1 \\ Guoguang Liu² \\ Yaqi Cai ${ }^{1}$
}

${ }^{1}$ Key Laboratory of Environmental Chemistry and Ecotoxicology,

Research Center for Eco-

Environmental Sciences,

Chinese Academy of Sciences,

P.O. Box 2871, Beijing 100085,

P.R. China

${ }^{2}$ Key Laboratory of Environmental

Science and Engineering of High

Education of Henan Province,

School of Environmental Science

and Chemistry, Henan Normal

University, Xinxiang, 453002,

Henan, P.R. China

\section{Sensitivity enhancement of chlorinated phenols by continuous flow liquid membrane extraction followed by capillary electrophoresis}

This paper describes a new analytical system, based on the combination of continuous flow liquid membrane extraction (CFLME) enrichment and capillary electrophoresis (CE) separation, for analysis of chlorinated phenols in water samples. Five chlorinated phenols including 3-chlorophenol (3CP), 4-chlorophenol (4CP) 2,4-dichlorophenol (DCP), 2,4,6-trichlorophenol (TCP), and pentachlorophenol (PCP) were separated by $\mathrm{CE}$ with Tris/sodium dihydrogen phosphate solution containing methanol $1 \%(v / v)$ as the run buffer. CFLME related parameters were investigated and optimal enrichment was obtained by using $0.3 \mathrm{~mol} \mathrm{~L}^{-1}$ Tris as acceptor and with a sample $\mathrm{pH} 5.0$, a sample flow rate of $4.0 \mathrm{~mL} \mathrm{~min}^{-1}$, and an enrichment sample volume of $150 \mathrm{~mL}$. The detection limit $(S / N=3)$ was $6.9,1.0$, and $1.7 \mathrm{ng} \mathrm{mL}^{-1}$ for DCP, PCP, and TCP, respectively. The reproducibility (RSD\%, $n=6)$ was 5.7 for DCP, 2.5 for PCP, and $2.8 \%$ for TCP $(n=6)$. The proposed method was applied to the determination of chlorinated phenols in spiked water samples with relatively satisfactory recoveries.

Key Words: CFLME; Capillary electrophoresis; Chlorinated phenols; Enrichment

Received: May 30, 2003; revised: August 22, 2003; accepted: February 18, 2004

DOI 10.1002/jssc.200301632

\section{Introduction}

In recent years capillary electrophoresis (CE) has become a powerful and widely used separation technique because, compared to liquid chromatography (LC), it provides relatively high resolving power, faster and more efficient separation, much lower reagent consumption, and similar detectability at trace levels [1].

However, because of its intrinsic poor concentration sensitivity, CE has hitherto gained very limited application in environmental analysis. Therefore, in order to enhance the sensitivity of $\mathrm{CE}$, many researchers have invested considerable effort in the development of this valuable technique and relevant achievements of great importance have been reported in the literature. These reported techniques can be divided into three main areas: one is sample enrichment on-column [2-5], another exploitation of detectors that can afford better sensitivity on hyphenation with CE [6-7], and the third one is sample pretreatment.

Sample pretreatment is relatively cheap, convenient, and easily performed. Therefore, many research reports dealing with this aspect can be found in the literature. Microdialysis [8], solid phase extraction (SPE) [9], and mem-

Correspondence: Guibin Jiang, Key Laboratory of Environmental Chemistry and Ecotoxicology, Research Center for Eco-Environmental Sciences, Chinese Academy of Sciences, P.O. Box 2871, Beijing 100085, China. Phone: +86 1062849334 .

Fax: +86 10 62849179. E-mail: gbjiang @ mail.rcees.ac.cn.

J. Sep. Sci. 2004, 27, 576-580

www.jss-journal.de brane extraction (SLM) [10-11] are among the widely used sample pretreatment methods.

Among the membrane extraction methods, the supported liquid membrane (SLM) technique is the one most extensively used; moreover, there have been reports that it can be coupled on-line with CE [11], The inherent drawbacks of SLM are the limited lifetime of the liquid membrane, the limited range of organic solvents suitable for use as liquid membrane, and the relatively low enrichment rate, which results in long enrichment times when very large enrichment factors are required. Our research group has developed a new liquid membrane extraction technique named continuous flow liquid membrane extraction (CFLME) for trace enrichment $[12,13]$. CFLME has intrinsic merits of high enrichment rate, long-term stability, and easy to select liquid membrane - in fact almost all polar organic solvents can be used as organic liquid membrane.

CFLME has been successfully coupled on-line to HPLC for analysis of sulfonylurea herbicides [12,13], and as it is mentioned above that SLM could be also successfully coupled with $\mathrm{CE}$, it should be feasible to couple CFLME online with CE because CFLME and SLM are similar procedures. However, the study of CFLME coupled to CE is still terra incognita. The goal of this paper was to investigate the possibility of such coupling.

Chlorinated phenols are present as pollutants in the aquatic environment as a result of the degradation of pesticides

(C) 2004 WILEY-VCH Verlag GmbH \& Co. KGaA, Weinheim 
and insecticides or the transformation of non-chlorinated phenols during chlorination of water. Due to their intrinsic toxicity and potential carcinogenicity, chlorinated phenols have been regarded as priority pollutants in the aquatic environment by the US Environmental Protection Agency (EPA) [14]. Chlorinated phenols were selected as model compounds in this present study. Separation of chlorinated phenols is not an easy task because substituted chlorinated phenols sometimes differ only very slightly, making their characters very similar, especially in the case of the isomers. Up to now, various separation methods have been reported for chlorinated phenols $[15,16]$. To our knowledge, as long as the separation buffer was properly selected the chlorinated phenols could be well separated. Hence, in this paper an attempt was also made to separate the selected chlorinated phenols with a new buffer solution.

\section{Materials and methods}

\subsection{Reagents}

3-Chlorophenol (3CP) and 4-chlorophenol (4CP) standard solutions (1.00 $\mathrm{mg} \mathrm{mL}^{-1}$ ) were purchased from the Institute for Reference Materials of SEPA (Beijing, China). 2,4Dichlorophenol (DCP), 2,4,6-trichlorophenol (TCP), and pentachlorophenol (PCP) (1.00 $\left.\mathrm{mg} \mathrm{mL}^{-1}\right)$ were obtained from the National Research Center for Reference Material (Beijing, China). Standard stock solution (200 $\mathrm{mg} \mathrm{L}^{-1}$ ) was prepared by dissolving standards in ultrapure water and stored at $4{ }^{\circ} \mathrm{C}$. Working solutions were obtained daily by appropriately diluting the stock solutions with ultrapure water. Methanol was purchased from Scharlau Chemie S.A. (gradient HPLC grade, Barcelona, Spain). Tris(hydroxymethyl)aminomethane (Tris, 99.9\%, Biochemical regent, AMRESCO, Solon, $\mathrm{OH}$, USA), dichloromethane, sodium dihydrogen phosphate, sodium hydroxide, and hydrochloric acid were all analytical grade unless otherwise stated. All water used was purified with a Milli-Q system (Millipore, Bedford, MA); ultrapure water filtered with a $0.45-\mu \mathrm{m}$ micropore membrane was used for washing processes in CE.

\subsection{Apparatus and procedure}

CE was performed using an $\mathrm{HP}^{3 \mathrm{D}}$ system (Agilent Technologies, USA) equipped with an on-column diode array detector (DAD). A detection wavelength of $220 \mathrm{~nm}$ and an experimental temperature thermostated to $25^{\circ} \mathrm{C}$ were used throughout. Uncoated narrow bore silica capillaries (Ruifeng Chromatographic Implements Ltd., Yongnian, Hebei, China) with an inner diameter of $100 \mu \mathrm{m}$, a total length of $60 \mathrm{~cm}$, and effective separation length of $51.5 \mathrm{~cm}$ were used. A new capillary was sequentially rinsed with methanol, ultrapure water, and $1 \mathrm{~mol} \mathrm{~L}^{-1}$ sodium hydroxide each for $10 \mathrm{~min}$. Before injection, the capillary was

J. Sep. Sci. 2004, 27, 576-580 www.jss-journal.de conditioned by sequential flushing with $0.1 \mathrm{~mol} \mathrm{~L}^{-1}$ sodium hydroxide, ultrapure water, and run buffer each for $10 \mathrm{~min}$. The capillary was rinsed with $0.1 \mathrm{~mol} \mathrm{~L}^{-1}$ sodium hydroxide, ultrapure water, and run buffer each for 5 min every five runs. Between runs the capillary was washed for $5 \mathrm{~min}$ with the separation electrolyte. Separation buffer was prepared by freshly mixing $0.2 \mathrm{~mol} \mathrm{~L}^{-1}$ Tris, sodium dihydrogen phosphate, and methanol at optimum concentrations as investigated in the following.

The CFLME system consists of a SLM extraction disk, two piston pumps, one peristaltic pump, and two six-port valves. The whole system setup is similar to those described elsewhere [12, 13], but a WZ-50G microinfusion pump (The Medical Instrument Factory of Zhejiang University, China) and a MiniPump (Laboratory Date Control, Division of Milton Roy Company) were employed to deliver dichloromethane and sample solution, respectively. The SLM extraction disk is the same as described elsewhere [17]. After a scheduled interval, the peristaltic pump was activated to transfer $2 \mathrm{~mL}$ of the acceptor containing enriched analytes into a graduated cuvette for determination by $\mathrm{CE}$.

\subsection{Sample preparation}

Aqueous samples of $10 \mathrm{ng} \mathrm{mL}^{-1} \mathrm{PCP}$ and TCP and $30 \mathrm{ng} \mathrm{mL}^{-1} \mathrm{DCP}$ were obtained by fortifying freshly filtered ultrapure water with stock solution to optimize operations.

Two real-world water samples, one collected from a local reservoir and the other drinking water from the market, were analyzed. These samples were filtered through a $0.45-\mu \mathrm{m}$ microporous membrane, and the same process was then following as for a standard solution as described above.

\section{Results and discussion}

\subsection{Electrophoretic separation of chlorinated phenols}

Chlorinated phenols have attracted more attention owing to their high toxicity, and methods for analyzing these compounds have been reported. Capillary zone electrophoresis, micellar electrokinetic chromatography, and nonaqueous capillary electrophoresis or hyphenated pretreatment techniques are often found in the literature. In most cases, phosphate-borate buffer and phosphate buffer were the chief running background buffer for separation. In the present case, phosphate and Tris were tried as the separation electrolyte. Since the buffer $\mathrm{pH}$ and organic additives would obviously influence the performance of separation, the effect of these parameters was investigated.

It was interesting that a change of buffer $\mathrm{pH}$ led to a change of peak position of PCP and TCP. TCP emerged

(C) 2004 WILEY-VCH Verlag GmbH \& Co. KGaA, Weinheim 


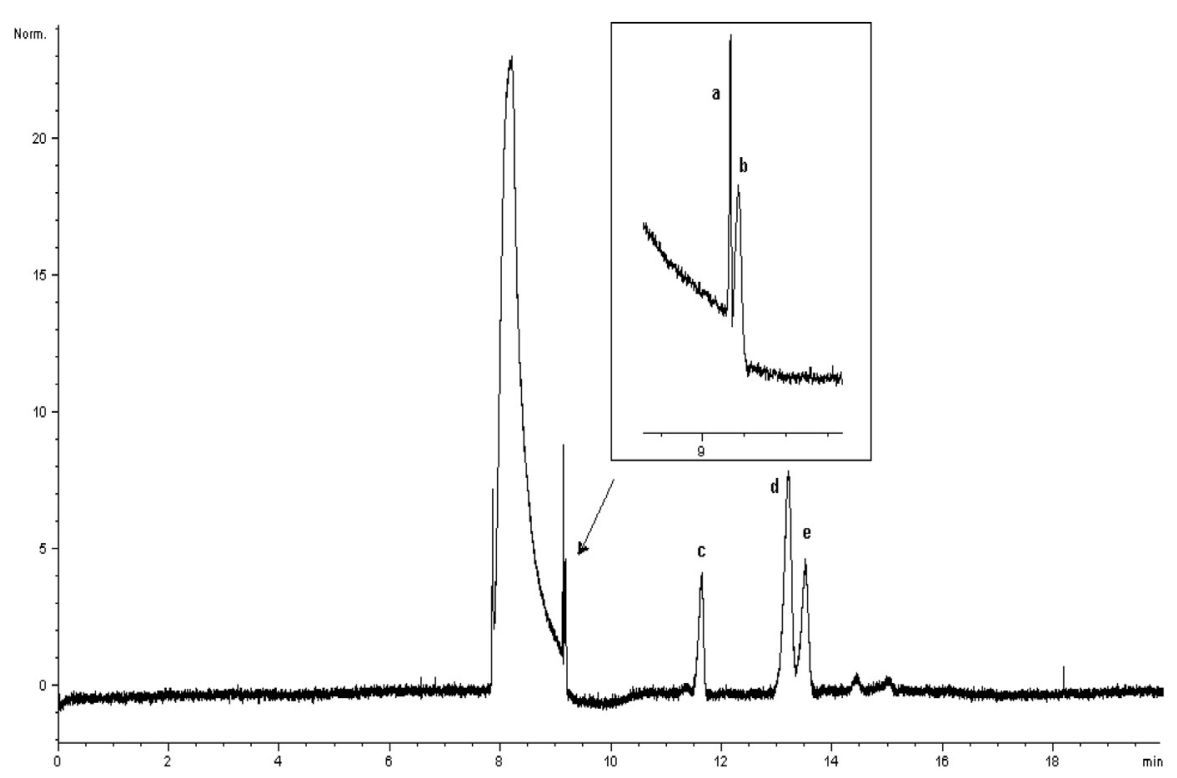

Figure 1. Electropherogram of five chlorinated phenols enriched with CFLME. a: 4-CP, b: 3-CP, c: DCP, d: PCP, e: TCP. Conditions: Injection pressure: 50 mbar, injection time: $10 \mathrm{~s}$, separation voltage: $10 \mathrm{kV}$, run buffer: contained Tris $17.2 \mathrm{mM}, 8 \mathrm{mM}$ sodium dihydrogen phosphate and $1 \%(\mathrm{v} / \mathrm{v})$ methanol. ahead of PCP in the acidic buffer, but behind of PCP in the neutral and weakly alkaline environment. The monosubstituted-chlorinated phenols (3-CP and 4-CP) could not be separated without organic additives. As a result, the importance of the presence of organic additive could not be ignored. In this study, four organic solvents including methanol, acetonitrile, acetone, and tetrahydrofuran at a concentration of $4 \%(\mathrm{v} / \mathrm{v})$ were investigated as a means of improving the resolution. Methanol and acetonitrile had almost the same ability for separating PCP and TCP, but methanol was better for separating monosubstitutedchlorinated phenols. In contrast, acetone and tetrahydrofuran could improve the separation of PCP and TCP, but showed a poor performance for 3-CP and 4-CP. Methanol was therefore selected as organic modifier, and the concentration was further optimized. Experiments demonstrated that the best separation electropherogram was obtained with a constant applied potential of $10 \mathrm{kV}$, buffer containing $17.2 \mathrm{mM}$ Tris and $8 \mathrm{mM}$ sodium dihydrogen phosphate and $1 \%(\mathrm{v} / \mathrm{v})$ methanol with a $\mathrm{pH}$ value of 8.2 , and pressure injection at 50 mbar for $10 \mathrm{~s}$.

Figure 1 shows the electrophoretic separation of the five chlorinated phenols after enrichment. As can be seen, 3$\mathrm{CP}$ and 4-CP enriched in Tris emerged near the peak of the matrix of acceptor and it was difficult to accurately analyze. Therefore, 3-CP and 4-CP were not included in the test compounds for further investigation.

\subsection{Optimization of CFLME operation}

\subsubsection{Organic solvent used as liquid membrane}

Organic solvent used as liquid membrane is a main factor that plays important roles in CFLME. An experiment showed that dichloromethane has a higher enrichment

J. Sep. Sci. 2004, 27, 576-580 www.jss-journal.de factor for polar sulfonylurea herbicides [12], which was in accordance with the principle of like dissolves like. Dichloromethane was chosen as liquid membrane because chlorinated phenols are polar compounds.

\subsubsection{Effect of acceptor phase and its concentration}

The selection of acceptors is very important in CFLME because whether the acceptor is optimum or not determines the extraction efficiency obtained in the extraction procedure. Generally speaking, an alkaline acceptor should be used for enrichment of weakly acidic compounds. Moreover, the acceptor should match the running buffer solution, otherwise analytes cannot be separated in the separation electrolyte. Four alkaline substances including $0.1 \mathrm{M} \mathrm{NaOH}, \mathrm{NaHCO}_{3}, \mathrm{Na}_{2} \mathrm{CO}_{3}$, and $\mathrm{Na}_{2} \mathrm{~B}_{4} \mathrm{O}_{7}$ were tried in selecting the optimal acceptor. The experimental results demonstrated that no peaks in electrophoretic analysis were obtained when $0.1 \mathrm{M} \mathrm{NaOH}$, $\mathrm{NaHCO}_{3}$, or $\mathrm{Na}_{2} \mathrm{CO}_{3}$ was used as acceptor phase, and only peaks of PCP and TCP in the electropherogram were obtained when $\mathrm{Na}_{2} \mathrm{~B}_{4} \mathrm{O}_{7}$ was used as acceptor phase. Tris is the only acceptor phase that can give all the analytes' peaks in electrophoretic analysis. This may be because $0.1 \mathrm{M} \mathrm{NaOH}, \mathrm{NaHCO}_{3}$, and $\mathrm{Na}_{2} \mathrm{CO}_{3}$ have a high ion strength which led to a low voltage drop in the sample zone when the separation voltage was applied. The analytes had a low mobility and could not move out from the acceptor matrix. This result indicates that the selection of acceptor must match the running buffer of CE if CFLME is hyphenated with $C E$.

The concentration of acceptor was further optimized. At low concentration of Tris, mono and disubstituted chlorinated phenols showed no peaks in the electropherogram,

(C) 2004 WILEY-VCH Verlag GmbH \& Co. KGaA, Weinheim 


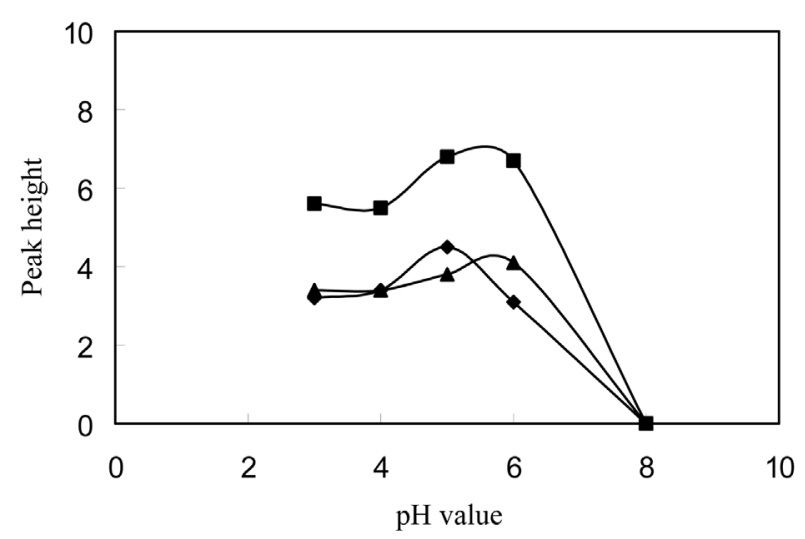

Figure 2. Effect of sample pH. (•) DCP, (•) PCP, (^) TCP.

while the peak heights of analytes decreased if the concentration of Tris was higher than $0.3 \mathrm{~mol} \mathrm{~L}^{-1}$. Consequently, $0.3 \mathrm{~mol} \mathrm{~L}^{-1}$ Tris was determined as the acceptor concentration for the experiments performed.

\subsubsection{Effect of sample solution $\mathrm{pH}$}

Chlorinated phenols are weak acidic and alkaline compounds, which must be able to turn into a neutral molecule form for the sake of being better trapped in dichloromethane. No doubt the acidity of the sample was one of the main complications in the enrichment. Sample $\mathrm{pH}$ was varied over the range of 3-8 by fortifying the working solution in freshly filtered ultrapure water with $2 \mathrm{~mol} \mathrm{~L}^{-1}$ or $0.1 \mathrm{~mol} \mathrm{~L}^{-1}$ hydrochloric acid to adjust the $\mathrm{pH}$ to the needed value. Extraction at ambient temperature with a flow rate of $3 \mathrm{~mL} \mathrm{~min}^{-1}$ was used to study the effect of $\mathrm{pH}$. Results shown in Figure 2 indicate that sample $\mathrm{pH}$ had a significant effect on the trapping of chlorinated phenols. No chlorinated phenols were found in the acceptor Tris when the sample was enriched at $\mathrm{pH}$ 8.0. After considering all aspects, a pH of 5.0 was adopted for the following study.

\subsubsection{Effect of flow rate}

With the constant $\mathrm{pH}$ value of 5.0 and same volume, the flow rate was optimized in the range of $1-5 \mathrm{~mL} \mathrm{~min}-1$. The result demonstrated that there was no significant change of peak heights of each chlorinated phenol over 1-4 $\mathrm{mL} \mathrm{min}^{-1}$, while the peak heights of the three chlorinated phenols decreased markedly at a flow rate of $5 \mathrm{~mL} \mathrm{~min}^{-1}$. Therefore, in order to save much time and keep a better trap performance, $4.0 \mathrm{~mL} \mathrm{~min}^{-1}$ was adopted in later experiments.

\subsubsection{Effect of sample volume}

Generally, the enrichment factor will be enlarged when a larger volume of sample is enriched. Results shown in Figure 3 indicate that different chlorinated phenols have different tendencies to change with changing enriched

J. Sep. Sci. 2004, 27, 576-580 www.jss-journal.de

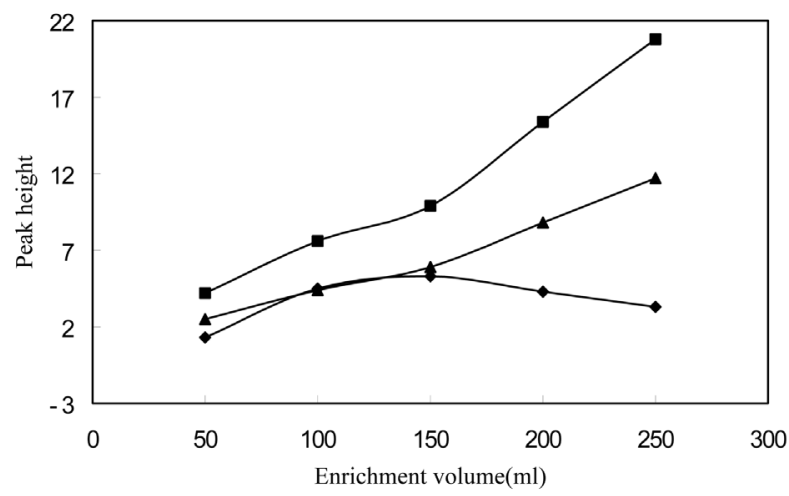

Figure 3. Effect of enriched volume. (•) DCP, (๘) PCP, (ム) TCP.

sample volume. The peak height of DCP increased with the sample volume in the range of $50-150 \mathrm{~mL}$, and then decreased with increasing sample volume. The peak height reached its maximum at a sample volume of about $150 \mathrm{~mL}$. Yet TCP and PCP demonstrated another tendency, showing a direct increase with increasing sample volume. This may be due to the fact that PCP and TCP have very low $\mathrm{p} K_{\mathrm{a}}$, they can be effectively enriched in acceptor. However, DCP has a relatively high $\mathrm{p} K_{\mathrm{a}}$ value which needs a relatively high acceptor $\mathrm{pH}$ for trapping analytes. Thus with increasing sample volume, more and more interferants will be trapped in acceptor and result in a decreasing acceptor $\mathrm{pH}$, which gives rise to a decreasing enrichment factor and even the already trapped analytes will back-extracted to the sample solution. Consequently, $150 \mathrm{~mL}$ was employed as the enrichment volume of sample.

\subsection{Calibration and detection limit}

Using the above-optimized experimental conditions, five standard solutions over the range of $20-60 \mathrm{ng} \mathrm{mL}^{-1}$ DCP, and 5-80 ng mL ${ }^{-1}$ PCP and TCP were analyzed for obtaining the linear range, detection limit and other characteristics of the method. The reproducibility was also evaluated by measuring a standard containing $5 \mathrm{ng} \mathrm{mL}^{-1}$ of PCP and TCP, and $20 \mathrm{ng} \mathrm{mL}^{-1}$ DCP over 6 runs. The peak area of chlorinated phenols was found to be linear over the whole range tested. The detection limit $(S / N=3)$ was $6.9,1.0$, and $1.7 \mathrm{ng} \mathrm{mL}^{-1}$ for $\mathrm{DCP}, \mathrm{PCP}$, and TCP, respectively. The reproducibility $(\mathrm{RSD} \%, n=6)$ was 5.7 , 2.5, and 2.8 for DCP, PCP, and TCP, respectively. These results showed that the detection limits of chlorinated phenols were satisfactory, but these were higher than the detection limits of the SLM-HPLC method developed by Knutsson et al. that were all lower than $0.1 \mu \mathrm{g} \mathrm{L}^{-1}$ [18]. It was attributed to the fact that $C E$ has poorer concentration sensitivity than HPLC and the ECD itself has a higher sensitivity than the UV detector.

(C) 2004 WILEY-VCH Verlag GmbH \& Co. KGaA, Weinheim 
Table 1. Mean recovery of real water spiked with concentrations of $20 \mathrm{ng} \mathrm{mL}^{-1}$ for DCP and $5 \mathrm{ng} \mathrm{mL}^{-1}$ for PCP and TCP.

\begin{tabular}{|l|c|c|c|}
\hline Water matrix & $\begin{array}{c}\text { Com- } \\
\text { pound }\end{array}$ & $\begin{array}{c}\text { Recovery } \\
{[\%]^{\mathrm{a})}}\end{array}$ & RSD [\%] \\
\hline Reservoir water & $\mathrm{DCP}$ & $98 \pm 7$ & 7.1 \\
\cline { 2 - 4 } & $\mathrm{PCP}$ & $112 \pm 12$ & 11.1 \\
\cline { 2 - 4 } & $\mathrm{TCP}$ & $63 \pm 4$ & 5.8 \\
\hline Drinking water & $\mathrm{DCP}$ & $104 \pm 9$ & 8.7 \\
\cline { 2 - 4 } & $\mathrm{PCP}$ & $99 \pm 3$ & 3 \\
\cline { 2 - 4 } & $\mathrm{TCP}$ & $104 \pm 4$ & 4.1 \\
\hline
\end{tabular}

a) Mean of four determinations \pm standard deviation.

\subsection{Analysis of spiked sample}

The method was applied to the determination of DCP, $\mathrm{PCP}$, and TCP in reservoir water and drinking water. It was observed that no chlorinated phenols were detected in the two water samples. These samples were then utilized as media for testing the possibility of the proposed method for determining chlorinated phenols in complex matrix. Table 1 shows the recovery and reproducibility of $5 \mathrm{ng} \mathrm{mL}^{-1}$ PCP, TCP and $20 \mathrm{ng} \mathrm{mL}^{-1}$ DCP spiked water samples. In these two water samples, the reproducibility of the three chlorinated phenols in the spiked water was in the range of $3-11.1 \%$, and the recoveries of DCP and PCP were in the range of $98-112 \%$, yet TCP had a better recovery in the drinking water of $104 \%$ and a lower recovery of $63 \%$ in the reservoir water. Maybe there was humic material in the reservoir water which could bind chlorinated phenols, thus accounting for the low recovery. However, the real cause leading to the low recovery of TCP in reservoir water calls for a thorough investigation and this drawback could be theoretically avoided.

\section{Concluding remarks}

A novel CFLME-CE system has been developed for the analysis of chlorinated phenols. The results demonstrate that it is possible to establish a hyphenated technique combining CFLME and CE on-line. In the developed method, chlorinated phenols were first preconcentrated by CFLME and then separated by capillary electrophoresis with a new buffer system, which consisted of Tris and sodium dihydrogen phosphate and methanol.

The proposed method provides satisfactory enrichment for chlorinated phenols. Moreover, it is inexpensive, con- venient, and easy to operate. The detection limit covered the range of $1-6.9 \mathrm{ng} \mathrm{mL}^{-1}$. The recoveries of three chlorinated phenols in two real water samples were in the range of $98-112 \%$ at $5 \mathrm{ng} \mathrm{mL}^{-1} \mathrm{PCP}$, TCP and $20 \mathrm{ng} \mathrm{mL}^{-1}$ DCP spiked level, except for a relatively low recovery of $63 \%$ for TCP in reservoir water. Potentially this method offers a more competitive alternative for environmental analysis.

\section{Acknowledgement}

This work was financially supported by the National Natural Science Foundation of China and the State High Tech Development Plan (20137010, 2001AA640610). The authors are grateful to Prof. J.Å. Jönsson for supplying SLM Extraction Disk and to Agilent Technologies for providing the $\mathrm{HP}^{3 \mathrm{D}} \mathrm{CE}$ system.

\section{References}

[1] N.A. Guzman, Capillary Electrophoresis Technology. Marcel Dekker, Inc., New York, NY, 1993, pp. iii-vi.

[2] M. Albert, L. Debusschere, C. Demesmay, J.L. Rocca, J. Chromatogr. A 1997, 757, 281-289.

[3] J.P. Quirino, Jong-Bok Kim, S. Terabe, J. Chromatogr. A 2002, 965, 357-373.

[4] Jong-Bok Kim, K. Otsuka, S. Terabe, J. Chromatogr. A 2001, 932, 129-137.

[5] J.P. Quirino, S. Terabe, Anal. Chem. 2000, 72, 1023-1030.

[6] M. Kölhed, P. Hinsmann, P. Svasek, J. Frank, B. Karlberg, B. Lendl, Anal. Chem. 2002, 73, 3843-3848.

[7] W.H. Chen, S.Y. Lin, C.Y. Liu, Anal. Chim. Acta 2000, 410, 25-35.

[8] M.W. Lada, R.T. Kennedy, J. Neurosci. Methods 1997, 72, 153-159.

[9] W. Qin, H. Wei, S.F. Li, J. Chromatogr. Sci. 2002, 40, 387391.

[10] S. Pálmarsdóttir, L. Mathiasson, J.Å. Jönsson, L.-E. Edholm, J. Chromatogr. B1997, 688, 127-134.

[11] S. Pálmarsdóttir, E. Thordarson, L.-E. Edholm, J.Å. Jönsson, L. Mathiasson, Anal. Chem. 1997, 69, 1732-1737.

[12] J.F. Liu, J.B. Chao, G.B. Jiang, Anal. Chim. Acta 2002, 455, 93-101.

[13] J.B. Chao, J.F. Liu, M.J. Wen, J.M. Liu, Y.Q. Cai, G.B. Jiang J. Chromatogr. A 2002, 955, 183-189.

[14] L.H. Keith, Compilation of Sampling Analysis Methods, US Environmental Protection Agency, Boca Raton, FL, 1991.

[15] S. Fu, S.G. Chu, X.B. Xiao, Chromatographia 2002, 56, 6972.

[16] K. Otsuka, S. Terabe, T. Ando, J. Chromatogr. A 1985, 348, 39-47.

[17] N. Megersa, J.Å. Jönsson, Analyst 1998, 123, 225-331.

[18] M. Knutsson, L. Mathiasson, J.Å. Jönsson, Chromatographia 1996, 42, 165-170. 